\title{
Vision Calibration of Mobile Robot
}

\author{
Mingqin Liu \\ College of mechanical and electrical engineering \\ China University of Mining and Technology \\ China \\ School of mechanical engineering \\ Huaihai Institute of Technology \\ China
}

\author{
Xiaoguang Zhang and Guiyun $\mathrm{Xu}$ \\ College of mechanical and electrical engineering \\ China University of Mining and Technology \\ China
}

\begin{abstract}
In consideration of robot's bumpy moving condition, a camera height dynamic compensation algorithm which based on characteristics of parallel lines was raised up, this camera height dynamic compensation algorithm is based on inherent characteristics that two parallel lines parallel to each other to conduct a dynamic compensation for the heights of robot vision camera. After comparing the calibration deviation of this algorithm with the calibration deviation of Opencv calibration algorithm, this algorithm showed a better result. According to the undulating road experiment result, the height which counted by this algorithm is closer to actual height than the height counted by other algorithm. Analysis and experiment results shows that this calibration compensation algorithm can significantly reduce calibration error which caused by undulating road.
\end{abstract}

Keywords-camera calibration; dynamic compensation; vision measurement; mobile robot

\section{INTRODUCTION}

Mobile robot processes and analyzes environment images in order to distinguish surrounding objects. In this process, mobile robot need recreate or distinguish targets according to camera captured images, therefore, calibrate the camera is essential. There are many foreign scholar's researches of monocular camera calibration, most of them using a fixed reference such as plane grid and three-dimensional grid to do calibration. For example, the ARGO system of the University of Parma, Italy [1] assumes that the camera internal parameters are known, then draws grids which have known dimensions on the ground, after that, it using grid intersections to calibrate external parameters. The position of those calibration points relative to the vehicle coordinate system is known. GRASP Laboratory of the University of Pennsylvania use straight lines which represent the traffic lane to do external parameters calibration, Song Xuefeng, etc.use the grids on ground to do calibration, through introduction of series image processing technologies such as edge extraction, Hough conversion, Vanishing Point detection, etc. to conduct the line detection of the grid-like ground images in both vertical and horizontal direction, then determine the location of calibration points, and finally achieved rapid calibration of the camera.

\section{THEORY}

There are four coordinate system involved in the camera's imaging geometry[2]: World coordinate system
$O_{V}-X v Y v Z v$,camera coordinate system $O_{C} X c Y c Z c$, image plane coordinate system $O-(u, v)$ and the pixel coordinate system $O_{1^{-}}(i, j)$.

Assume the ground is flat, camera coordinate system origin is on the camera's optical center, direction of the optical axis is $X c$, roll angle relative to the robot is $\varphi$ (the clockwise tilt which observed along the direction of robot's travel is positive), pitch angle is $\theta$ (upward is positive), Orientation angle is $\psi$ (point to the left side of robot's travel direction is positive), in world coordinate system, the coordinate of the optical center $t=(l, b, h)$, if there is a point $(p)$ in world coordinate system, its coordinate is $p_{v}\left(x_{v}, y_{v}, z_{v}\right)$, its coordinate in the camera coordinate system is $p_{c}\left(x_{c}, y_{c}, z_{c}\right)$, through rotation and translation transformation, the coordinate transformation between $p_{c}\left(x_{c}, y_{c}, z_{c}\right)$ and $p_{v}\left(x_{v}, y_{v}, z_{v}\right)$ can be created.

$$
\begin{array}{r}
p_{v}^{T}=R \cdot p_{c}^{T}+t^{T} \text { or } \\
p_{c}^{T}=R^{-1} \cdot p_{v}^{T}-R^{-1} \cdot t^{T}=R^{T} \cdot p_{v}^{T}-R^{T} \cdot t^{T}
\end{array}
$$

Among them , $\mathrm{R}$ is rotation matrix, $\mathrm{t}$ is translation matrix(vector).

For line L, which is on the flat ground surface and parallel to longitudinal axis of robot body $\mathrm{Xv}$, and the distance from the line $\mathrm{L}$ to $\mathrm{Xv}$ is a, the parametric equations of the line $\mathrm{L}$ in robot vehicle coordinate system can be expressed as:

$$
x_{v}=s, y_{v}=a, z_{v}=0
$$

$s$ is any real number. Below is the transformation relation between the image plane coordinate system and pixel coordinates:

$$
\left\{\begin{array}{l}
u=\left(i-c_{i}\right) d_{x} \\
v=\left(j-c_{j}\right) d_{y}
\end{array}\right.
$$

Among the formula, $d_{x}, d_{y}$ are respectively represent the effective distance between two adjacent pixels (mm / pixel) in the horizontal direction ( $\mathrm{x}$ direction) and in the vertical direction (y-direction); ( $c i, c j)$ are the coordinates of the 
image coordinate origin in pixel coordinates valueis (in pixels); $u, v$ is the coordinate in image plane coordinate system; $i, j$ is the coordinate in pixel coordinate system.

For three parallel lines $l_{1} 、 l_{2} 、 l_{3}$ which parallel to $X_{\mathrm{v}}$, their vanishing point in pixel coordinate system is $\left(i_{h}, j_{h}\right)$, the coordinate of any point on each line is $\left(i_{n}, j_{n}\right)$, their slopes in pixel coordinate system can be written as :

$$
k_{n}=\left(j_{h}-j_{n}\right) /\left(i_{h}-i_{n}\right) \quad(\mathrm{n}=1,2,3)
$$

Take formula (3) into (4), we can obtain:

$$
\begin{aligned}
& k_{n}^{\prime}=d u / d v=\left(u_{h}-u_{n}\right) /\left(v_{h}-v_{n}\right)=k_{n} d_{x} / d_{y}= \\
& \left(d_{x} f_{i} / d_{y} f_{j}\right) \cdot\left(a_{n} r_{12} r_{21}-d r_{12} r_{21}-h r_{12} r_{31}-a_{n} r_{11} r_{22}+d r_{11} r_{22}+h r_{11} r_{32}\right) \\
& /\left(a_{n} r_{13} r_{21}-d r_{13} r_{21}-h r_{13} r_{31}-a_{n} r_{11} r_{23}+d r_{11} r_{23}+h r_{11} r_{33}\right)
\end{aligned}
$$

Make $r_{n}=-\left(f_{j} d_{y} k_{n}^{\prime}\right) /\left(f_{i} d x\right)=-f_{j} k_{n} / f_{i}$,

Take (1) into (7), then solve the simultaneous equations, the result is:

$$
\psi=\operatorname{arctg}\left\{\frac{\left(r_{1}-r_{3}\right)\left(a_{1}-a_{2}\right)-\left(r_{1}-r_{2}\right)\left(a_{1}-a_{3}\right)}{\left(r_{1}-r_{3}\right)\left(r_{1} a_{1}-r_{2} a_{2}\right)-\left(r_{1}-r_{2}\right)\left(r_{1} a_{1}-r_{3} a_{3}\right)}\right\}
$$

According to formula (5)、(6) and (8), we can get

$$
\begin{aligned}
& \theta=\operatorname{arctg}\left[\left(u_{h} \sin \psi / f_{i} d_{x}\right)+\left(v_{h} \cos \psi / f_{j} d_{y}\right)\right] \\
& \varphi=\operatorname{arctg}\left\{\left[\cos \theta\left(u_{h} / f_{i} d_{x}\right)-\sin \psi t g \theta\right] / \cos \psi\right\}
\end{aligned}
$$

When the camera is fixed to the robot body, $1, \mathrm{~b}$ is almost no change during robot's moving process, so these two parameters can be considered as fixed, we can measure these two parameters after assembling the camera onto robot body, but since $\mathrm{h}$ is a dynamic parameter vary with robot's bumpy moving condition, so we need to conduct dynamic measurement during travelling process of robot.

The detailed measuring method is: Pick any straight line from pixel coordinate system which parallel to pixel axis i, this line have two intersections $(a, b)$ with any two lines which parallel to $\mathrm{XV}$ in images, in world coordinate system, these two intersections correspond to point $\mathrm{A}$ and $\mathrm{B}$, the distance between point $\mathrm{A}$ and $\mathrm{B}$ is $\mathrm{d}$, shown as figure 1 .

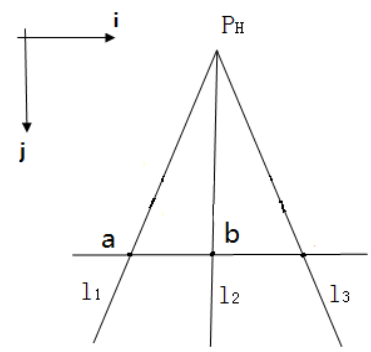

(a)

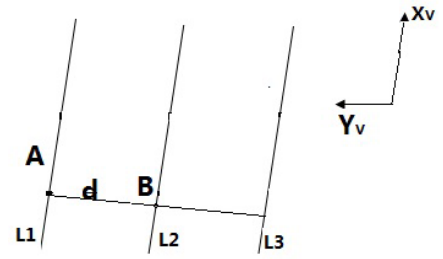

(b)

(a)in pixel coordinate system (b) in world coordinate system

FIGURE I. A STRAIGHT LINE AND THREE PARALLEL LINES INTERSECT

Proved by theory and experiment, in pixel coordinate system, $\mathrm{j}_{\mathrm{a}}=\mathrm{j}_{\mathrm{b}}$, but in world coordinate system,

Assume that[6]

$$
x_{a v} \neq x_{b v}
$$

In image coordinate system, the line slope is:

$$
k_{n}^{\prime}=d u / d v=\left(u_{h}-u_{n}\right) /\left(v_{h}-v_{n}\right)=k_{n} d_{x} / d_{y}
$$

According to formula (3) and (6), we can get $k_{n}{ }^{\prime}$ 


$$
\left.d=\sqrt{\left(y_{a v}-y_{b v}\right)^{2}+\left(x_{a v}-x_{b v}\right)^{2}}=\sqrt{\left[h\left(p_{a}-p_{b}\right) /\left(r_{31}+p_{b} r_{32}+q_{b} r_{33}\right)\left(r_{31}+p_{2}+p_{a} r_{32}+q_{22}\left(r_{31}+q r_{33}\right)\right] \cdot\right]} \cdot\right]
$$

When assembling the camera in a correct way, the value of $\theta . \psi \cdot \varphi$ is very small, so,

$$
\sqrt{\left[r_{32}\left(r_{21}+r_{23} q\right)-r_{22}\left(r_{31}+q r_{33}\right)\right]^{2}+\left[r_{32}\left(r_{11}+r_{13} q\right)-r_{12}\left(r_{31}+q r_{33}\right)\right]^{2}} \approx q
$$

$$
d=y_{a v}-y_{b v}=h\left(p_{a}-p_{b}\right) q /\left[\left(r_{31}+p_{b} r_{32}+q_{b} r_{33}\right)\left(r_{31}+p_{a} r_{32}+q_{a} r_{33}\right)\right]
$$

Then

$h=d\left[M^{2}+M(\cos \theta \sin \phi)\left(p_{a}+p_{b}\right)+p_{a} p_{b}\left(\cos ^{2} \theta \sin ^{2} \phi\right)\right] /\left[\left(p_{a}-p_{b}\right) q\right]$

Among them,

$$
M=r_{31}+q r_{33}=\sin \theta+q \cos \theta \cos \phi, q=q_{a}=q_{b}
$$

According to above formula, when the value of $d$ is known, take the calculated value of $\theta 、 \psi 、 \varphi$ into formula (15),we can get the value of $h$. Based on the value of $h$ which derived by formula (15), and combining other calculating processes of parameter calibration, we can obtain the camera external parameter $\mathrm{h}$ which can adapt to the dynamic changes in the external environment. So, we can carry out accurate transformation between world coordinate system and the camera coordinate system accordingly.

\section{METHOD}

\section{A. Calculate the Value of $\theta . \Psi$. $\varphi$ in Rotation Matrix}

Calculate the value of $\theta, \psi, \varphi$ in rotation matrix is conducted in three situations, shown as figure 2to4: In the first situation, there are 3 obvious parallel lines and an obvious vanishing point of those 3 parallel lines exist in captured images. The pixel of vanishing point $P_{H}\left(u_{h}, v_{h}\right)$ is obviously displayed on the image, in this situation, we just need to pick total three points from each of those 3 lines as $P_{1}\left(i_{1}, j_{1}\right) 、 P_{2}$

$\left(i_{2}, j_{2}\right), P_{3}\left(i_{3}, j_{3}\right)$, and measure the distance from each of three lines(or road lane or track line of sports field) to $X_{V}$ of world coordinate system $a_{1}, a_{2}, a_{3}$, then take above parameters and camera internal parameters $f i, f j, d x, d y(f i, f j$ are respectively represent the focal length in $i$ direction and $j$ direction(in pixels); $d x, d y$ are respectively represent the horizontal and vertical scale coefficient) into formula (8)、(9) and (10), we can calculate the value of $\theta, \psi, \varphi$, and get the rotation matrix $R$. In the second situation, there are not obvious vanishing point of three parallel lines, in such a situation, we can pick up each two points from each of those three lines, total six points are $P_{1}^{\prime}, P_{1}^{\prime \prime}, P_{2}^{\prime}, P_{2}^{\prime \prime}, P_{3}^{\prime}, P_{3}^{\prime \prime}$, then calculate the gradient of those three lines $\left(k_{n}\right)$.

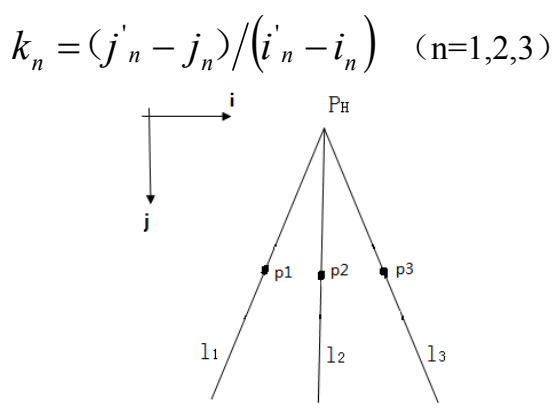

(a)

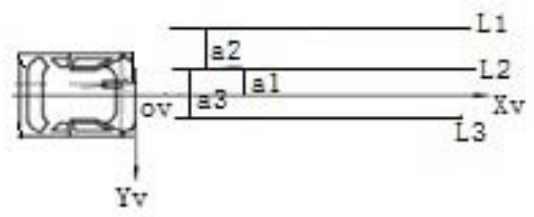

(b)

(a)in pixel coordinate system. (b) in world coordinate system FIGURE II. THE FIRST SITUATION
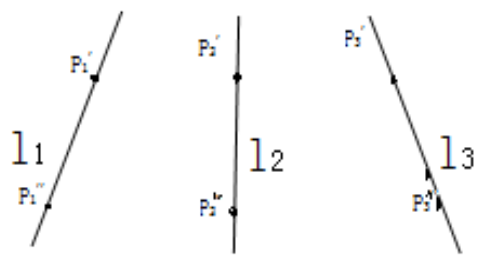

FIGURE III. THE SECOND SITUATION

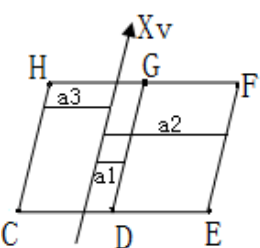

(a)

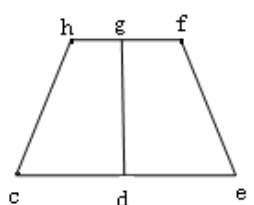

(b) (a) in pixel coordinate system.

(b) in world coordinate system

FIGURE IV. THE THIRD SITUATION

Based on above, we can work out the point-gradient formula of those three straight lines.

$$
j-j_{n}=k_{n}\left(i-i_{n}\right) \quad(\mathrm{n}=1,2,3)
$$

According to the formula(3)、(6 - 10) 、(16), we can calculate the value of $\theta, \psi, \varphi$ and obtain the rotation matrix $R$.

In the third situation, there are no obvious parallel lines on 
the captured images, in such a situation, we can select six points which are distributed as a double rectangle, the six points are listed as $\mathrm{C} 、 \mathrm{D} 、 \mathrm{E} 、 \mathrm{~F} 、 \mathrm{G} 、 \mathrm{H}$, and the line of DG、 $\mathrm{EF} 、 \mathrm{CH}$ are consistent with the direction of $\mathrm{XV}$, their distance from each of DG,EF and $\mathrm{CH}$ to XV are $a_{1}, a_{2}, a_{3}$, we can find corresponding six points from pixel coordinate system as $c 、 d$ 、 e、f、g、 h, at this time, three lines dg, ef, ch meet the calculation condition of the second situation, so, we can use the second calculation method to obtain the value of $\theta 、 \psi 、 \varphi$.

\section{B. Calculation of the Value of $H$}

If there are obvious parallel lines on the captured images, we can pick up a straight line which parallel to pixel axis $i$ in pixel coordinate system, then assume this line is intersected with another two parallel lines, find the intersection $a, b$ as shown on figure 5.If there is no any obvious parallel line on the captured images, we can conduct calculation according to the third situation, in this situation, we can pick up one straight line which parallel to pixel axis $i$ and intersect with any two of $c h, d g, e f$ in the pixel coordinate system, then, find the pixel coordinate of the intersection, take them into formula (1)、(3)and (10) to calculate the value of $P, P$ and $q$, then based on the points of $a_{1}, a_{2}, a_{3}$ to calculate the distance $d$ of two parallel lines which intersect with the line we picked up from the pixel coordinate system. Take the value of $d$ into formula (6), we can get $h$.

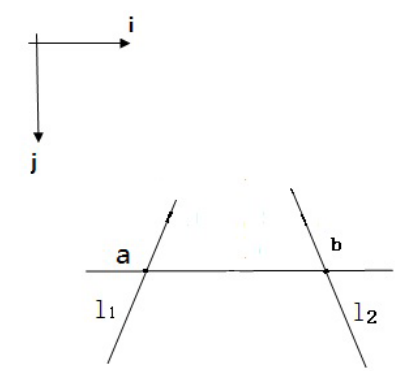

FIGURE V. THERE ARE OBVIOUS PARALLEL LINES ON THE CAPTURED IMAGES

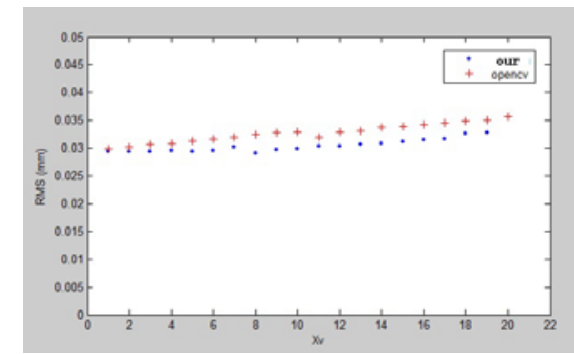

FIGURE VI. RMS BY OUR METHOD AND OPENCV METHOD

\section{CALIBRATION EXPERIMENT AND DEVIATION ANALYSIS}

\section{A. Calibration Experiment}

The calculated camera external parameters by method which provided [3] is compared with the calculated parameters by above proposed method. When calculated using the above proposed algorithm, the chosen images have five characteristics, they are: (a) there are three obvious parallel lines and a obvious vanishing point on the captured images; (b) there are three obvious parallel lines but no vanishing point on the captured images;(c) there are two obvious parallel lines and an obvious vanishing point on the captured images;(d) there are two obvious parallel lines but no vanishing point on the captured images;(e) there in no any parallel lines on the captured images. We respectively calculate the $\theta, \psi, \varphi, h$ in each of five images through above method in this article, then compare the rotation vector $R$ and translation vector $T$ with the result which calculated by Opencv method. In this experiment, the camera internal parameters is calibrated by toolbox_calib which provided by Matlab[4], after calibration, we can get the camera main point $\left(c_{i}, c_{j}\right)$ and the effective focal length $f_{i}, f_{j}$ 。 The $d_{x}, d_{y}$ can be obtained by lens parameters. We use a same camera in this experiment, $\mathrm{CCD}$ is $8 \mathrm{~mm} * 6 \mathrm{~mm}$, resolution ratio is $640 * 480$ pixel, then $d_{x}=d_{y}=1 / 80(\mathrm{~mm} / \mathrm{pixel})$. When use Opencv method to do calculation, the method is to use a same cameras and carrier,move standard checkerboard images in the same scene, and continuously capture 20 images.

\section{B. Deviation Analysis}

For verifying the correctness of above method, after calculated rotation matrix $R$ and translation matrix $T$, we picked up a group of feature points which the pixel coordinate and corresponding world coordinate of those points are known. We use rotation matrix $R$ and translation matrix $T$, what separately calculated by this article proposed method and Opencv, to reverse those points's data in world coordinate system. Then use those results and those feature points's real world coordinate to mean square deviation as the index of evaluation of measuring accuracy[5]. The formula is listed below:

$$
R M S=\sqrt{\left(X_{W}-X_{W 0}\right)^{2}+\left(Y_{W}-Y_{W 0}\right)^{2}+\left(Z_{W}-Z_{W 0}\right)^{2}} / 3
$$

In the formula(17), RMS is the mean square deviation of the recovery point; $\left(X_{w}, Y_{w}, Z_{w}\right)$ is the feature points's world coordinate which is calculated by this article proposed method and Opencv method. $\left(X_{w 0}, Y_{w 0}, Z_{w 0}\right)$ is the feature points's real world coordinates. Calculating data is listed in figure 6.

The selected feature points are distributed from near to far along the $X_{V}$ axis, total 20 points. The horizontal axis is $X_{V}$ coordinate, vertical axis is $R M S$, the calculation result is from (b) (there are three obvious parallel lines but no vanishing point on the captured images).The result by Opencv method are respectively marked in the figure 6 . It shows that, with the increase of $X_{V}, R M S$ value has an increasing trend. Meanwhile, the $R M S$ increasing trend by this article proposed method is more gentle than the increasing trend by Opencv.

\section{CONCLUSION}

According to camera perspective projection principle, three parallel lines have the same vanishing point and different slope on the image plane, there is an intrinsic relation exist between the external parameters of robot's camera, the vanishing point and the slopes. We can deduce the analytical expression of the external parameters of the robot vision camera, we can implement compensation for the camera height by using the inherent characteristics of parallel lines. Experiment result shows, this article proposed method can 
accurately calibrate the effective height of the camera in undulating road condition. Comparing with some present methods, this method have many advantages such as simple principle, easy operation, strong versatility, diverse implementations, easy to be integrated in the robot vision system. After introducing the image processing technology, the whole calibration process can be carried out automatically, can even be carried out in a moving robot, thus, this method has in certain extent solved the drift problem of robot vision camera parameters.

\section{ACKNOWLEDGEMENT}

The sduty carried out with the support of Huaihai Institute of Techology Natural Science Foundation(Z2012002).

\section{REFERENCES}

[1] Bertozzi M,Broggi A., A parallel real-time stereo vision system for generic obstacle and lane detection[J]. IEEE Transactions on Image Processing, 1998, 7(1) 62-81.

[2] Li Qing,Zheng Nan-ning,Zhang Xue-tao,Calibration of external parameters of vehicle-mounted camera with trilinear method. Opto-Electronic Engineering, 2004,31(8),23-26.

[3] Chen Shengyong, Liu Sheng. Realization of Computer Vision Technology Based on Opencv . Science Press, Beijing, 2008,pp.364-365.

[4]

http://www.mathworks.cn/videos/image-processing-with-matlab-82545. html.

[5] NiuHaitao,ZhaoXunjie,New method of camera calibration based on checkerboard, Infrared and Laser Engineering .2011,40(1),133-137. 